\title{
GEOSPATIAL SERVICE PLATFORM FOR EDUCATION AND RESEARCH
}

\author{
Jianya Gong, Huayi Wu, Wanshou Jiang, Wei Guo, Xi Zhai, Peng Yue
}

\author{
State Key Laboratory of Information Engineering in Surveying, Mapping and Remote Sensing (LIESMARS), \\ Wuhan University, 129 Luoyu Road, Wuhan, 430079, China \\ Email: gongjy@whu.edu.cn
}

\section{Commission VI}

KEY WORDS: Geospatial Web Service, Geoprocessing, Service Chaining, Education

\begin{abstract}
:
We propose to advance the scientific understanding through applications of geospatial service platforms, which can help students and researchers investigate various scientific problems in a Web-based environment with online tools and services. The platform also offers capabilities for sharing data, algorithm, and problem-solving knowledge. To fulfil this goal, the paper introduces a new course, named "Geospatial Service Platform for Education and Research", to be held in the ISPRS summer school in May 2014 at Wuhan University, China. The course will share cutting-edge achievements of a geospatial service platform with students from different countries, and train them with online tools from the platform for geospatial data processing and scientific research. The content of the course includes the basic concepts of geospatial Web services, service-oriented architecture, geoprocessing modelling and chaining, and problem-solving using geospatial services. In particular, the course will offer a geospatial service platform for handson practice. There will be three kinds of exercises in the course: geoprocessing algorithm sharing through service development, geoprocessing modelling through service chaining, and online geospatial analysis using geospatial services. Students can choose one of them, depending on their interests and background. Existing geoprocessing services from OpenRS and GeoPW will be introduced. The summer course offers two service chaining tools, GeoChaining and GeoJModelBuilder, as instances to explain specifically the method for building service chains in view of different demands. After this course, students can learn how to use online service platforms for geospatial resource sharing and problem-solving.
\end{abstract}

\section{INTRODUCTION}

Geospatial service platforms play an important role in the areas of analysing geospatial data, sharing data, algorithm, and problem-solving knowledge. As geospatial services technology has matured in recent years, a growing amount of Web resources and processing functions are available in the form of Web services (Gong et al., 2009; Zhao et al., 2012). For example, a past project funded by 2009 ISPRS Scientific Initiative has provided near two hundred geoprocessing services for geoscientific research (Yue et al., 2010). Although millions of people across the world are interacting with geospatial data via online tools such as virtual globes or online maps, few people has chances to utilize online geoprocessing services for their education and research. A geospatial service platform, equipped with computing facilities and analysis functions, can support domain analyses of geospatial data in a Web-based environment. Since scientific users are often lack of knowledge of Web programming and geospatial Web services, it is then necessary to develop an appropriate course to share cuttingedge achievements of a geospatial service platform with students and researchers from different countries, thus promoting CyberInfrastructure-based research and education.

This paper presents a new course, named "Geospatial Service Platform for Education and Research", which will be delivered in the ISPRS Summer School to be held by State Key Lab of Information Engineering in Surveying, Mapping and Remote Sensing (LIESMARS), in May 2014, at Wuhan University, China. It will be held in conjunction with 2014 Mid-Term Symposium ISPRS Commission VI - "Data, Information, and Knowledge Sharing for Geo-Education" (ISPRS 2014). The course will introduce the basic knowledge and recent progress of geospatial services, advanced geospatial service tools and platforms. The course will train students to solve complex scientific problems using geospatial services. Students will select one of three types of hands-on practices, depending on their background knowledge in Web programming. The three kinds of practices are geoprocessing algorithm sharing through service development, geoprocessing modelling through service chaining, and online geospatial analysis using geospatial services. For example, if students are interested with Web programming, they can learn the basic concepts of geospatial services by wrapping their geoprocessing algorithm as Web Services. Existing geoprocessing services from two open service toolkits, OpenRS and GeoPW, will be introduced. If students are interested in using geoprocessing functions only, they can discover and invoke online geoprocessing services directly, or learn how to do complex geoprocessing using geoprocessing modelling. In the geoprocessing modelling, geoprocessing services are chained as scientific workflows and executed in a workflow engine. There are two service chaining tools, GeoChaining and GeoJModelBuilder, in this course. The former one is implemented using BPEL (Business Process Execution Language), and executed in a BPEL-based workflow engine. The later one is a light-weight, open-source model builder, having its own workflow description and execution engine. Finally, an application system - GeoSquare is introduced to students, where they can find data and analysis functions, conduct analysis, and check analysis results in a Web environment.

The remainder of this paper is organized as follows: Section 2 presents the knowledge content of the course; Section 
3 describes exercises; Section 4 provides a syllabus for the course; Conclusions are given in Section 5.

\section{KNOWLEDGE CONTENT OF THE COURSE}

The availability of massive high-resolution Earth observation data such as remote sensing images enables the exploration of complex geographical phenomena (Gong et al., 2009). However, the data is so big that it is far more than Earth scientists' capabilities to analyze. In scientific problem solving, the capability to carry out geospatial analysis is essential. Domain experts are familiar with the geoprocessing processes and remote sensing algorithms. Once they learn to provide algorithms as services, or use services provided by others, the capabilities in scientific problem solving could be improved significantly. For example, supposing Mr. Zhai is an expert in remote sensing, he wants to know the situation of flood inundation in Poyang Lake in China. He can get MODIS images from an online data service. Then he can process each image by invoking the Normalized Difference Vegetation Index (NDVI) calculation, binarization, and rendering services to generate a thematic flood map that shows the coverage of the flood around the Poyang Lake (Sun et al., 2012).

Service-Oriented Architecture (SOA), a distributed information architecture, has shown great potential in scientific research. It provides a way that reorganizing a portfolio of previously siloed software applications through public interface and protocol. The standards from SOA allow service systems interoperable, extensible, and reusable. Service modules from different providers can be shared and support the distributed information infrastructure. In the SOA, there are three roles, service provider, service broker, and service requester. These roles allow researchers to publish, find, and bind geospatial resources in an interoperable environment. Web Services are a set of technologies for the implementation of SOA. Major standards for Web Services include SOAP (Simple Object Access Protocol) and WSDL (Web Services Description Language), defined by W3C (World Wide Web Consortium). In addition to SOAP-based Web Services, light-weight Web Services following the style of REpresentational State Transfer (REST), named RESTful Web Services (Pautasso et al., 2008), are also available.

In the geospatial domain, the Open Geospatial Consortium (OGC) has defined a series of specifications for geospatial Web Services, such as Web Feature Service (WFS), Web Map Service (WMS), Web Coverage Service (WCS), Catalogue Service for the Web (CSW), Web Processing Service (WPS), and sensor services (Sensor Observation Service, Sensor Planning Service, Sensor Event Service, and Web notification service) (OGC, 2007). Some open source implementation of these service specifications are also available (52North, 2012). In a geospatial Web environment, geospatial data and various analysis functions can be Web-accessible through these services. These services provide a flexible and effective way to manage and share geospatial Web recourses.

The growing amount of geospatial services shows great potential to solve complex geoprocessing problems in a distributed environment. Each geoprocessing service, as a basic functional analysis unit, can be combined together to fulfil a complex geoprocessing task. ModelBuilder tools are needed to help users visually combine different analysis processes and generate geoprocessing models. Geoprocessing services together with data and sensor services are often chained as scientific workflows and executed in workflow engines (Sun et al., 2012). The workflow can be executed using available geoprocessing services. The summer course offers two service chaining tools, GeoChaining and GeoJModelBuilder. The tools can allow users to drag and drop various geospatial services to visually generate workflows and interact with the workflows in a virtual globe environment (Zhang et al., 2013). It also allows users to audit trails of workflow executions, support scientific reproducibility, and check the provenance of data products.

In the course, several service tools will be introduced as follows:

- $\quad \mathrm{GeoPW}$ is a set of services providing geoprocessing functions over the Web. The goal of GeoPW is to address the geoprocessing demands in the emerging information infrastructure. It was funded by the 2009 ISPRS Science Initiative, "Integrating Virtual Globe and Web Service Technologies for ISPRS Higher-Education Teaching and Research". The services in GeoPW are implemented by developing middleware that wraps legacy GIS analysis components to provide a large number of geoprocessing utilities over the Web. They continue to operate after the end of the project. These services are open and accessible to the public, and they support integrated geoprocessing on the Web. Near two hundred geoprocessing services are available now in GeoPW for geoscientific research.

The OpenRS initiative aims to build an open interoperable geoprocessing framework primarily for remote sensing imagery (Gong et al., 2009). The goal of OpenRS is to implement highly extensible, scalable, customizable, and configurable remote sensing image processing toolbox that enables the development of advanced geoprocessing functions or applications. The OpenRS framework encompasses basic image processing operations such as image mapping, image enhancement, georeferencing, and classification. The entire OpenRS framework includes four layers: core plug-in management (orsBase), service plug-ins, object plug-ins, and application programs. A set of standard development interfaces is defined to facilitate the development of various plug-ins that are highly interoperable with each other. Cloud-enablement services can be implemented through a dedicated distributed programming model and a distributedfile system. Web-based graphic interfaces allow interactive parallel job monitoring.

GeoChaining is implemented using BPEL (Business Process Execution Language), and executed in a BPELbased workflow engine. It supports advanced and complex chaining modes and seamlessly connects to a Data \& Service Registration Center and incorporates GeoGlobe as built-in visualization environments. All registered geospatial resources can be readily accessed in GeoChaining, in which quality information of resources is also available. With GeoGlobe visualization capabilities, users can directly observe remote geospatial resources (e.g. data, geoprocessing, and WMS service) as well asfinal execution results of GIService chains.

GeoJModelBuilder is a light-weight, open-source model builder, having its own workflow description and execution engine. It is a java implementation of ModelDriven approach for geoprocessing workflows. It couples geoprocessing Web Services, NASA World Wind and Sensor Web Services to support geoprocessing modeling and environmental monitoring. The tool can allow users to drag and drop various geospatial services to visually generate workflows and interact with the workflows in a virtual globe environment. It also allows users to audit trails of workflow executions, check the provenance of data products, and support scientific reproducibility. 
GeoJModelBuilder (Figure 1) is available at http://sourceforge.net/projects/geopw/.

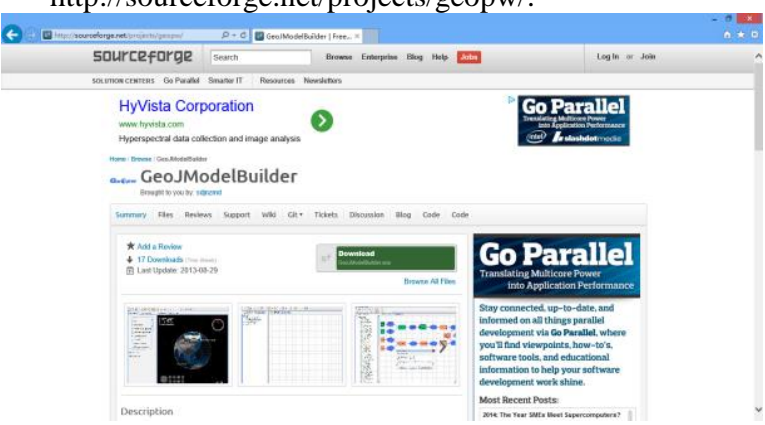

Figure 1. GeoJModelBuilder - an open source modelling tool.

The GeoSquare project was implemented based on common Web technologies such as the enterprise Web portal, Rich Internet Applications, Java User Interface toolkits, and workflow engines. Most of these technologies are built upon open source standards and easy to develop. These technologies ensure the delivery of content-rich and cross-platform applications. In addition, the compliance of Web Services and OGC standards significantly promote GeoSquare interoperability. GeoSquare integrates complex service chaining tools (GeoChaining), open high-performance geoprocessing platform (OpenRS), and three-dimensional visualization clients (GeoGlobe) into an integrated environment. GeoSquare (http://www.geosquare.org) is capable of manipulating Internet-based GIServices and other geospatial resources with easy-to-use user interfaces (Gong et al., 2009).

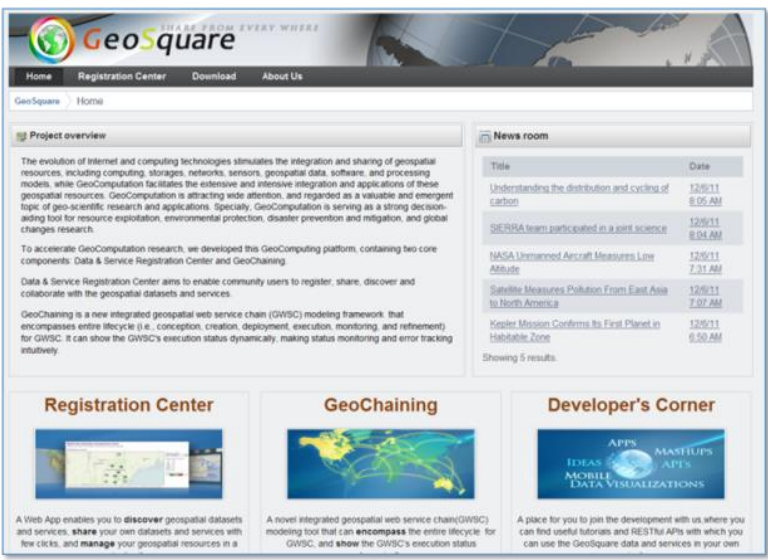

Figure 2. The user interface of GeoSquare.

In addition, the course will also introduce the cloud computing - Microsoft Azure in delivering geospatial cloud services. Microsoft Azure was first announced in 2008. After that, a developer version was freely available for public tests. The formal version was published at the beginning of the year 2010. The platform provides a Cloud environment to help developers build, host, and scale applications through Microsoft data centers. It provides a platform for running Windows applications and storing their data in the Cloud. Windows Azure runs on a large number of servers in Microsoft data centers, which are connected into a unified whole by Windows Azure Fabric. Windows-based compute and storage services for Cloud applications are built on top of this fabric. Geoprocessing functions can be deployed in Microsoft Azure to provide more scalable and reliable services (Yue et al., 2013).

\section{EXERCISES}

The course will develop three kinds of exercises, shown as follows.

Exercise 1: Practicing the basic concepts of geospatial services by wrapping their geoprocessing algorithm as a Web Services. The OpenRS is used to share models and algorithms in an interoperable Web environment. OpenRS offers a oneclick service wrapping function, shown in Figure 5. Using the executable object model of OpenRS, it can automatically generate executable object invoking agent. The operation of executable object invocation is transformed into a task submitted to the distributed parallel processing platform (Guo et al., 2010).

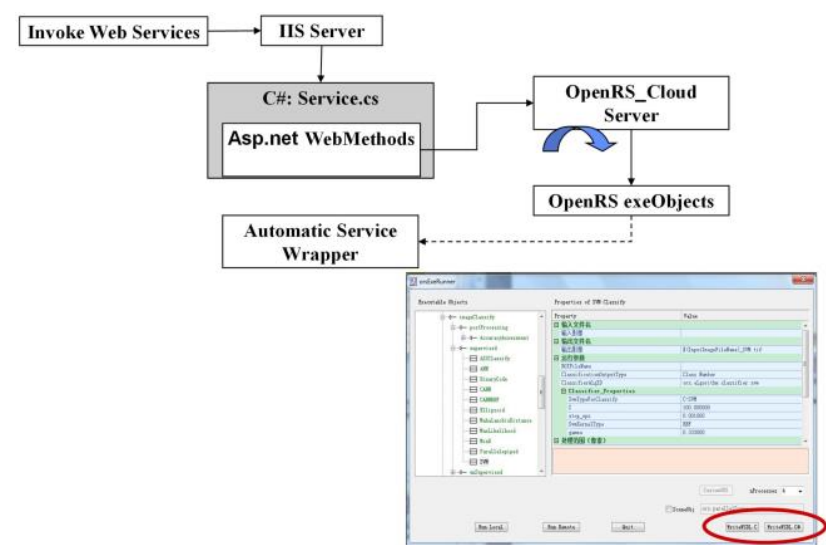

Figure 3. Wrapping algorithm as a Web service using OpenRS.

Exercise 2: Exercising geoprocessing modelling. In the geoprocessing modelling, geoprocessing services are chained as scientific workflows and executed in a workflow engine. The example on flood inundation analysis in Section 2 is used to help understand service chaining tools performed using distributed data and various geoprocessing services. Figure 4 (a) shows how the RasterMapcalcProcess service, RasterBinaryProcess service and RasterColorsProcess service are combined into a workflow model in sequence. The output of one service is connected to the input of the next service in the workflow. Figure 4 (b) and Figure 4 (c) show the modelling interfaces in GeoJModelBuilder and GeoChaining respectively. The final processing result of images (Figure 4 (d) and Figure 4 (e)) in this case can be visualized in different spatial data service platforms, such as TIANDITU (TIANDITU, 2014) or Google Map. 


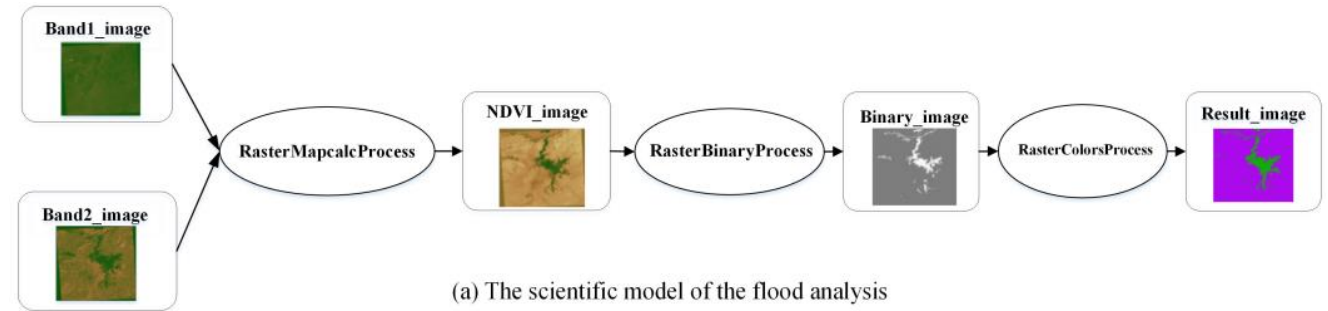

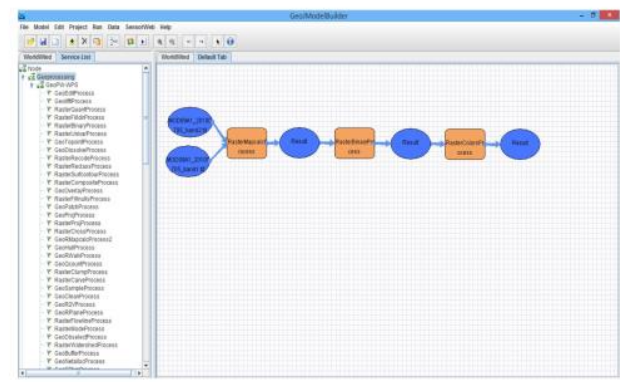

(b) Modelling Window in GeoJModelBuilder

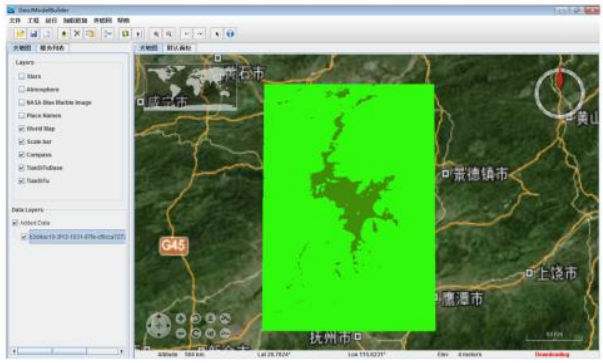

(d) Result from GeoJModelBuilder

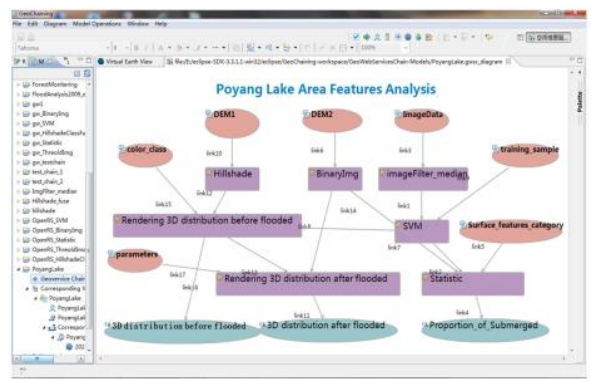

(c) Modelling Window in GeoChaining

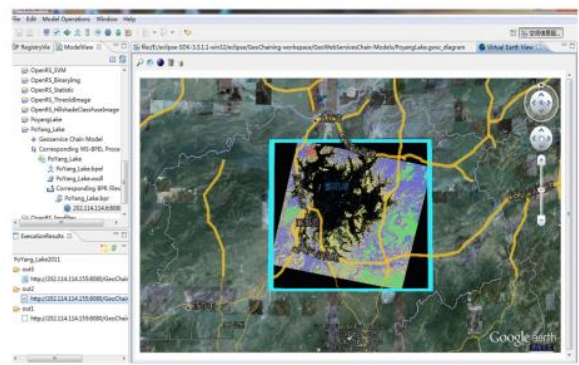

(e) Result from GeoChaining

Figure 4. The geoprocessing modelling on flood analysis in GeoJModelBuilder and GeoChaining.

Exercise 3: Exercising the online analysis using existing geospatial services. If students are with the background of remote sensing and not skilled in Web Service technologies, they can go directly to an online analysis Web system GeoSquare. GeoSquare runs in Web browsers and works as a Web application. Students can find data and analysis services, conduct analysis by using research agenda of GSW, and check analysis results in a Web environment. Figure 5 shows the operating steps that can be followed in the Execise 3.

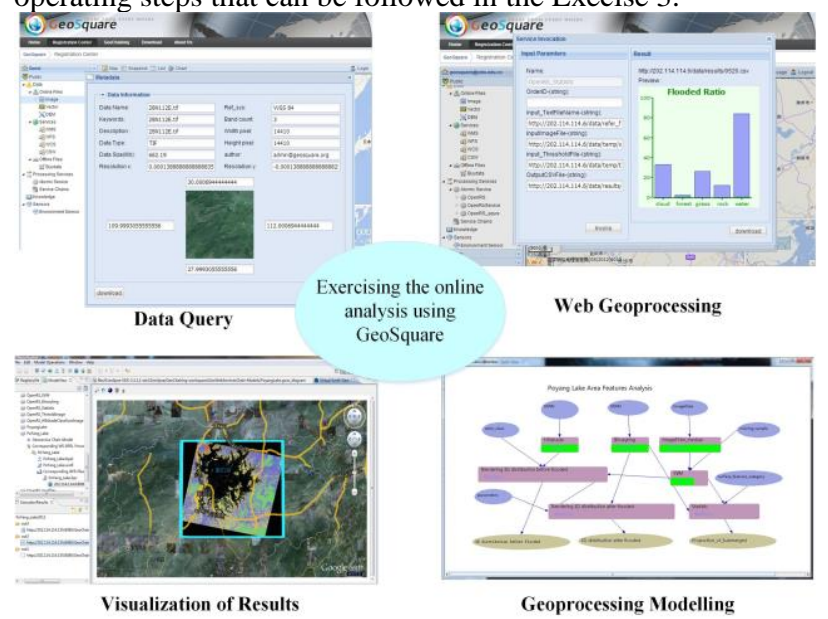

Figure 5. Exercising the online annlysis using GeoSquare.

We have launched a geospatial resource sharing platform for this course. Students can conduct various operations, including registration, query, update, and deletion of geospatial service, workflow, and data resources, through the platform. Furthermore, students can set up diverse groups according to the different interests and comment on the shared resources. Other users can invoke contributions available in the platform and make evaluation, thus it helps improve popularity and credibility of providers. The platform can register different types of geospatial services, including Web Services following W3C standards, and geoprocessing services and sensor Web services following the OGC specifications. Users can check the metadata information of a specific service, such as names, functions, and providers, in an intuitive Web form in the platform. Once different services are chained as workflows, the workflows can also be published in the platform as a kind of geospatial resources. Users can download any workflow package in the platform, and open it using the GeoJModelBuilder. Figure 6 illustrates workflows published in this platform. 


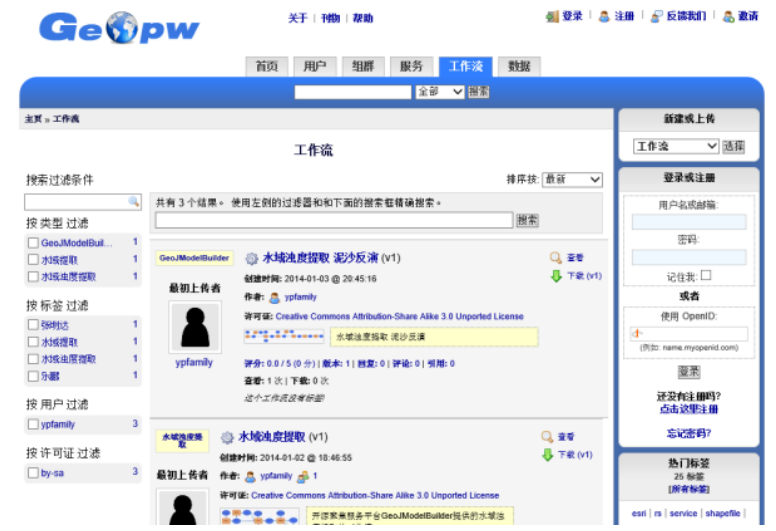

Figure 6. Workflow resources in the Web platform.

\section{SYLLABUS OF THE COURSE}

The course includes a set of frontal lectures, demo, and hands-on practices. The syllabus is arranged according to the content of the course to advance the understanding of geospatial service platforms. Lectures will be combined with hand-on practices. Background knowledge and existing geospatial service platforms described in section 2, such as GeoPW, OpenRS, GeoJModelBuilder, GeoChaining, GeoSquare, will be taught and practiced. Students can select one of the three kinds of exercises listed in Section 3. There will be practice manuals with detailed process description, cases, and example data before exercise. In the end, students will finish their group projects using skilled learnt from this course and present their projects. The detailed syllabus is shown in Table 1.

\begin{tabular}{|c|c|}
\hline \multicolumn{2}{|r|}{ Geoprocessing Services In Microsoft Azure } \\
\hline 1. & Introduce Microsoft's Cloud Platform-Windows Azure \\
\hline 2. & $\begin{array}{l}\text { Hands-on practice for Windows Azure } \\
\text { Students select interested project by themselves according } \\
\text { to the three kinds of exercises }\end{array}$ \\
\hline \multicolumn{2}{|r|}{ Background Knowledge and OpenRS } \\
\hline 3. & $\begin{array}{l}\text { Introduce background knowledge } \\
>\text { Geospatial Web services } \\
>\text { Service-oriented architecture } \\
>\text { Geoprocessing modelling and chaining } \\
>\text { Problem-solving using geospatial services }\end{array}$ \\
\hline 4. & - Introduce geoprocessing services platform OpenRS \\
\hline \multicolumn{2}{|c|}{ Geospatial Service Development and Geoprocessing Modelling (Part 1) } \\
\hline 5. & $\begin{array}{l}\text { Geoprocessing algorithm sharing through service } \\
\text { development } \\
\text { - Geoprocessing modelling through service chaining. }\end{array}$ \\
\hline \multicolumn{2}{|c|}{ Geoprocessing Using Services, Geoprocessing Modelling (Part 2) } \\
\hline 6. & $\begin{array}{l}\text { Online geospatial analysis using geospatial services } \\
\text { - Geoprocessing modelling through service chaining. }\end{array}$ \\
\hline \multicolumn{2}{|r|}{ Exercises } \\
\hline 7. & Exercises \\
\hline 8. & Project reports \\
\hline
\end{tabular}

Table 1. Syllabus of the course.

\section{CONCLUSIONS}

This paper introduces a new course, named "Geospatial Service Platform for Education and Research", to share cuttingedge achievements of a geospatial service platform with students and researchers from different countries. Knowledge on geospatial Web Services, service-oriented architecture, geoprocessing modelling and chaining, and problem-solving using geospatial services will be shared with students. It will be a "hands on" course, with many small exercises on using geospatial Web platform, such as GeoPW, OpenRS, GeoJModelBuilder, GeoChaining, GeoSquare, and Windows Azure. There will be three kinds of exercises in the course to advance the scientific understanding through applications of geospatial service platforms. Students can learn how to use geospatial service platforms for the problem-solving in this course.

\section{ACKNOWLEDGEMENTS}

This research is supported by National Basic Research Program of China (2011CB707105), National Natural Science Foundation of China (41271397), and Program for New Century Excellent Talents in University (NCET-13-0435).

\section{REFERENCES}

52North, 2012. 52North Sensor Web Community, http://52north.org/communities/sensorweb/, (Accessed 19 March, 2011).

Guo, W., Gong, J., Jiang, W., Liu, Y., She, B., 2010. OpenRSCloud: A remote sensing image processing platform based on cloud computing environment. Science China Technological Sciences, 53(1), pp.221-230.

Gong, J., Wu, H., Gao, W., Yue, P., Zhu, X., 2009. Geospatial Service Web. In Li, D., Shan, J., Gong, J. (eds) Geospatial 
Technology for Earth Observation Data. Springer New York, Dordrechet Heidelberg London, pp.355-379.

ISPRS, 2014. Mid-Term Symposium ISPRS Commission VI"Data, Information, and Knowledge Sharing for GeoEducation".http://www.lmars.whu.edu.cn/isprscom6/index. html, (Accessed 17 March, 2014).

OGC, 2007. OGC Sensor Web Enablement Architecture. Version 1.1.0. OGC 06-021r4. OGC Sensor Web Enablement ArchitectureOpen Geospatial Consortium, Inc. 2007.

Pautasso, C., Zimmermann, O., \& Leymann, F. (2008). Restful web services vs. big web services: making the right architectural decision. In Proceedings of the 17th international conference on World Wide Web (pp. 805814). ACM.
Sun, Z., Yue, P., Di, L., 2012. GeoPWTManager: a taskoriented web geoprocessing system. Computers \& Geosciences, 47, pp.34-45.

TIANDITU, 2014. http://www.tianditu.cn, (Accessed 24 March, 2014).

Yue, P., Gong, J., Di, L., Yuan, J., Sun, L., Sun, Z., Wang, Q., 2010. GeoPW: Laying blocks for the geospatial processing web. Transactions in GIS, 14(6), pp.755-772.

Yue, P., Zhou, H., Gong, J., Hu, L., 2013. Geoprocessing in Cloud Computing platforms-a comparative analysis. International Journal of Digital Earth, 6(4), pp.404-425.

Zhang, M., and Yue, P., 2013. GeoJModelBuilder: A java implementation of model-driven approach for geoprocessing workflows, In: 2013 Second International Conference on Agro-Geoinformatics (AgroGeoinformatics), IEEE publication, 5 pp, 2013.

Zhao, P., Foerster, T., Yue, P., 2012. The geoprocessing web. Computers \& Geosciences, 47, pp.3-12. 